\title{
Análisis de las competencias núcleo y el desarrollo empresarial en las MIPES: estudio de caso *
}

\author{
Analysis of Core Competencies and Business Development of SMEs: A Case \\ Study
}

Omar Neme-Castillo

Doctor en Ciencias económicas, Instituto Politécnico Nacional,

Ciudad de México-México, oneme@ipn.mx

\section{Ana Lilia Valderrama-Santibáñez}

Doctora en Ciencias económicas, Instituto Politécnico Nacional, Ciudad de México-México, avalderrama@ipn.mx

\section{Cesaire Chiatchoua}

Doctor en Ciencias económicas, Instituto Politécnico Nacional, Ciudad de México-México, chiatchoua@yahoo.co.uk

\section{Cómo citar / How to cite}

Neme-Castillo, O.; Valderrama-Santibáñez, A. L.; Chiatchoua, C. (2021). Análisis de las competencias núcleo y el desarrollo empresarial en las MIPES: estudio de caso. Revista CEA, v. 7, n. 13, e1599.

https://doi.org/10.22430/24223182.1599

Recibido: 19 de julio de 2020

Aceptado: 30 de octubre de 2020

\section{Resumen}

El documento identifica las competencias núcleo, entendidas como base de la ventaja competitiva, de una muestra de micro y pequeñas empresas de Iztapalapa en la Ciudad de México. Se sigue una doble metodología. Primero, se evalúan atributos de unicidad, colectividad y flexibilidad estratégica de las capacidades; después, se estima un modelo de corte transversal para determinar su contribución en el desarrollo empresarial. Para el sector industrial se establecen a los procesos y compras como competencias núcleo y ventas y marketing para los servicios. Se estima que estas competencias contribuyen a mantener bajos costos, a un rápido ajuste y al aumento de ventas e ingresos en el sector industrial y a la reducción de efectos negativos externos, implicando una pronta

\footnotetext{
*Este artículo se deriva del proyecto titulado «Diagnóstico de competencias organizacionales de los individuos en las micro y pequeñas empresas para el desarrollo productivo en la delegación Iztapalapa de la Ciudad de México» y ha sido financiado con recursos del Instituto Politécnico Nacional (SIP20150244).
} 
respuesta y un incremento de ventas e ingresos en el sector servicios. Los resultados sientan bases para el diseño de políticas de fomento de este grupo de empresas con carácter sectorial a través del impulso de competencias núcleo. Es necesario el diseño de programas de desarrollo de procesos de producción para empresas industriales con la esperanza de que configuren estructuras de costos por debajo del promedio. También es indispensable el diseño de programas de competencias en ventas y marketing para el sector de los servicios que minimicen los efectos negativos de competidores de mayor tamaño.

Palabras clave: ventajas competitivas, planificación estratégica, estrategias para el desarrollo, estrategia comercial, política de ventas.

Clasificación JEL: L11, M1, L25.

\begin{abstract}
In this paper, we identify the core competencies, which are the basis for competitive advantage, in a sample of Small and Medium-Sized Enterprises (SMEs) in Iztapalapa, Mexico City. For that purpose, we implemented a two-stage methodology. First, we evaluated the uniqueness, collectiveness, and strategic flexibility of the competencies. Second, we estimated a cross-sectional model to determine their contribution to business development. In the industrial sector, processes and purchases are core competencies; in turn, in the service sector, it is sales and marketing. We estimate that these competencies contribute to maintaining low costs, a quick adjustment, and an increase in sales and income in the industrial sector. In addition, they help to reduce negative external effects, which implies a fast response and an increase in sales and income in the service sector. These results lay the foundations for making policies that promote these enterprises by boosting their core competencies depending on their sector. Industrial enterprises should design programs to develop production processes and configure below-average cost structures. In turn, the service sector should design programs to develop sales and marketing skills that minimize the negative effects of bigger competitors.
\end{abstract}

Keywords: Competitive advantages, strategic planning, development strategies, commercial strategy, sales policy.

JEL classification: L11, M1, L25.

\title{
1. INTRODUCCIÓN
}

En un mundo globalizado y competitivo las fortalezas y debilidades de cada región o localidad pueden traducirse en mecanismos impulsores u obstaculizadores de su propio desarrollo económico (Chen et al., 2020). Sobre esta base, las potencialidades territoriales y las competencias núcleo (core competencies) de las empresas ahí ubicadas representan una ventaja competitiva sostenible para participar con éxito en el mercado. En el ámbito territorial, las competencias núcleo se entienden como una red integrada de capacidades (humanas, materiales y financieras) orientadas al desarrollo de ventajas competitivas dada la unicidad de operar en un lugar particular (Francioli \& Albanese, 2017). En este sentido, propiciar las competencias núcleo resulta fundamental para optimizar a las economías locales y alcanzar mayores estándares de vida de la población. 
Si se parte de este contexto, el desarrollo productivo depende de las core competencies dada alguna potencialidad del territorio; es decir, las empresas emplean sus ventajas internas en combinación con los recursos que ofrece el territorio local para mejorar la eficiencia en términos de costo, distribución, uso de materias primas y tiempos fortaleciendo sus ventajas competitivas y, en última instancia, su propio desarrollo (Ali, 2017). Para Castro (2010) las estrategias competitivas que desarrollan las empresas son un factor fundamental para saber la forma en que estas van a competir en los mercados y de ellas dependerá si se tiene o no éxito en su gestión empresarial. De este modo, cuando las empresas siguen estrategias a partir de sus competencias núcleo tienden a alcanzar estándares de desempeño más altos, contribuyendo al desarrollo económico de las localidades en donde se asientan (Huang et al., 2019).

De acuerdo con el Instituto Nacional de Estadística y Geografía (INEGI, 2016) del total de empresas consideradas, $97.6 \%$ son microempresas y concentran el $75.4 \%$ del personal ocupado total. Le siguen las empresas pequeñas, que son un $2 \%$ y tienen el $13.5 \%$ del personal ocupado. Las medianas representan $0.4 \%$ de las unidades económicas y tienen poco más del $11 \%$ de los ocupados. Entonces las Micro y Pequeñas (MIPES) representan $99.7 \%$ del total de las empresas en el país. En la Ciudad de México (CDMX), el total de unidades económicas al 2017 fue de 471,811 (9.3\% del total nacional), de las cuales $98.1 \%$ son micro o pequeñas empresas (INEGI, 2019). En el caso concreto de Iztapalapa, la Alcaldía de mayor población de la CDMX, es sede del mayor número empresas de la ciudad (prácticamente 1 de cada 5 empresas) con la mayor representatividad de MIPES al concentrar $17.4 \%$ del total en CDMX. Aún más, se aprecia una especialización en actividades terciarias, principalmente en comercio al por mayor y una relativa importancia de empresas de servicios de apoyo al productor (INEGI, 2017). No obstante, de acuerdo con la Universidad Nacional Autónoma de México (UNAM, 2011) se caracteriza por bajos niveles de productividad que contrastan con los registrados en otras demarcaciones, de hecho, Iztapalapa es la segunda Alcaldía con el mayor porcentaje de población que percibe ingresos inferiores a los dos salarios mínimos, perfilándola como una zona de bajos ingresos y rezago socioeconómico.

La heterogeneidad socioeconómica sugiere la existencia de factores de localización que determinan el desempeño de los agentes productivos dentro de este territorio y, por ende, impactan en sus competencias núcleo y competitividad. Existen aspectos sociodemográficos (población, densidad, vivienda), económicos (terciarización de las economías), culturales (tradiciones y fiestas) e históricos (prehispánica y colonial) que contribuyen a su unicidad local y configuran un perfil de individuos y empresas que inciden en las ventajas competitivas de esta localidad -dadas las relaciones sociales que se construyen al interior- y colocan a las MIPES en el centro de los modelos de producción socioterritorial a partir de sus competencias núcleo. Díaz (2003) presenta una idea similar respecto a los factores del desarrollo productivo.

Derivado de lo anterior, surge la pregunta principal del documento, ¿cuál es el efecto de las competencias núcleo de las MIPES en Iztapalapa sobre el desempeño empresarial, dado el carácter territorial específico de esta? Así, se plantea como hipótesis que las core competencies empresariales afectan positivamente este desempeño. El objetivo es doble. Primero, identificar las competencias núcleo de las micro y pequeñas empresas en Iztapalapa. Segundo, estimar la contribución de estas competencias en el desempeño empresarial de dichas MIPES. Como se reconoce en el trabajo este estudio contribuye a la limitada literatura que estudia los elementos que favorecen la competitividad 
de las MIPES desde la óptica de las competencias núcleo por ejemplo (Molina, 2009; Cantú Mata et al., 2012; Molina Sánchez et al., 2013; Sánchez Aguirre et al., 2017).

Además, el documento se compone de cinco partes. Junto a la introducción, se analiza la revisión de la literatura de las competencias núcleo, se identifican las competencias núcleo de las MIPES locales, se presentan la metodología, los resultados, la discusión y las conclusiones.

\section{MARCO TEÓRICO}

\section{Competencias núcleo}

Si bien no hay consenso sobre la definición de las competencias núcleo, los distintos acercamientos a su concepción siguen la misma línea de argumentación. Por ejemplo, para Prahalad y Hamel (1990) las competencias núcleo constituyen un stock de habilidades y tecnología acumulada a través del aprendizaje que contribuye a la ventaja competitiva de la empresa. De acuerdo con Williams (1992) son habilidades que permiten a las empresas ofrecer productos con beneficios para los clientes a través del establecimiento, mejora y reconfiguración de recursos que llevan a una ventaja competitiva sostenible. Hafeez et al., (2002) señalan que son recursos de la empresa consistentes en activos intelectuales, físicos y culturales y que se comprenden como algo único, especial, difícil de imitar y altamente competitivo. Sadler (2003) agrega que ninguna habilidad o rasgo cognitivo representa una competencia núcleo si no lleva a la empresa, directa o indirectamente, a una ventaja competitiva persistente mediante una mejor satisfacción de las necesidades de los clientes que los competidores.

Asimismo, una competencia central para las operaciones del negocio pero que no es excepcional en alguna forma para la industria, no es considerada como competencia núcleo, puesto que no representa un diferenciador de la empresa (Senthilkumar et al., 2013). De igual forma, Prahalad y Hamel (1990) establecen que los recursos estandarizados o de fácil acceso no propician que la empresa alcance una ventaja competitiva sobre sus rivales. No obstante, por medio de inversiones simultáneas y combinadas en activos intangibles, como conocimiento, habilidades, tecnología, procesos, imagen, relaciones, redes, entre otros, es posible desarrollar una competencia núcleo para una empresa en particular (Andriessen et al., 1999).

Hafeez et al., (2002) proponen que en la determinación de las capacidades clave es necesario aislar las capacidades clave del resto de capacidades mediante la evaluación de dos dimensiones: «colectividad» y «atributo único». La «colectividad» se entiende que es la flexibilidad operativa para la empresa y se evalúa al medir la integración: a través de funciones, a través de negocios y a través de operaciones de producción. Es decir, la integración de las capacidades clave en las actividades de negocio de toda la compañía. El «atributo único» evalúa cada capacidad clave integrada contra los tres atributos de singularidad a saber: rareza, imperfectamente imitables e insustituibilidad a través de una escala de medición. Finalmente determina la capacidad básica a través de la flexibilidad estratégica, la que se define mediante dos elementos: la redistribución de recursos y rutinas de reorganización. Por ejemplo, la minimización del capital de trabajo en el proceso de producción. Cuando los recursos de las empresas cumplen con estas características representan competencias núcleo (Clardy, 2007). 
En línea con lo anterior, las competencias núcleo se caracterizan por tres atributos básicos (Prahalad \& Hamel, 1990): colectividad, unicidad y flexibilidad estratégica, que se consideran en este estudio. La colectividad hace que las competencias empresariales sean de uso generalizado en la organización al generar sinergias entre las distintas actividades (Grant, 1991) y facilitar la entrada a nuevos segmentos de mercado o el desarrollo de productos. Para Prahalad y Hamel (1990), tres propiedades representan la colectividad: i) entre-productos, las competencias no son capacidades aisladas, sino plataformas para familias de productos que integran activos y habilidades (Bakker et al., 1994); ii) entre-funciones, las competencias se crean a través de esfuerzos integrados de equipos o miembros de la organización en distintas áreas o funciones (Grant, 1991); y, iii) al interior de la empresa (entreunidades empresariales), actuando en todas las áreas funcionales de la empresa representando la identidad propia de esta. Este rasgo es particularmente importante para empresas con negocios múltiples (Moingeon et al., 1998).

En cuanto a la unicidad, una capacidad única es un mecanismo "aislante» que puede evitar que los competidores erosionen la ventaja competitiva creada (Reed \& De Fillippi, 1990). Para ser «única», una capacidad debe mostrar al menos uno de los siguientes atributos (Prahalad \& Hamel, 1994): i) rara en el mercado, derivado de la experiencia específica de la empresa y la habilidad de acumular activos centrales, lo que permite establecer estrategias de creación de valor basadas en esta rareza (Grant, 1991); ii) no imitable; la imitabilidad se refiere al grado en que los recursos o capacidades de una empresa no pueden ser duplicados por sus competidores (Barney, 1991), en consecuencia, si son difíciles de imitar adquieren un valor adicional en el mercado; y, iii) no sustituible, si un recurso o capacidad son poco sustituibles es más probable que mantengan su superioridad en la generación de valor para la empresa.

Por último, la flexibilidad estratégica se entiende como la capacidad de una competencia de crear diferentes opciones para responder a las demandas de ambientes dinámicos (Volberda, 1996; Herhausen et al., 2020). Es la facilidad con la que los recursos clave de una competencia pueden implementarse para desarrollar nuevas capacidades. La flexibilidad consiste en dos atributos (Sánchez, 1995): i) redespliegue de recursos: si una competencia puede explotar sus recursos subyacentes e implementar opciones de forma flexible, esto es, desplegar los activos en múltiples aplicaciones para innovar (Goold et al., 1997) y, ii) reorganización de rutinas, la facilidad con la que las rutinas pueden reorganizarse para crear valor. Si bien una rutina puede ser valiosa en un periodo específico, puede crear una inercia organizacional que limita la capacidad de la empresa para comprender las nuevas señales del entorno, por ende, una rutina valiosa debe ser capaz de reorganizarse sistemáticamente y enfrentar las nuevas condiciones competitivas (Helleloid \& Simonin, 1994).

Así, las competencias son aquellas capacidades clave que son altamente colectivas en la operación empresarial y relativamente únicas en el mercado. La diferencia entre competencias y competencias núcleo es que las últimas son además estratégicamente flexibles (Zhang, 1999). Álvarez y Durán, 2009; Dini y Stumpo, 2018, proponen un amplio rango de capacidades generales de las MIPES, traslapadas algunas, a saber: innovación, segmentación, procesos tecnológicos, producción a gran escala, ingeniería de procesos, ensamblado, experimentación, administración de la producción, gerenciamiento del producto, servicio al cliente, fijación de precios, promoción, distribución, desarrollo de productos, investigación, investigación de mercados, administración del conocimiento, calidad, planeación financiera, ventas, fidelización, publicidad, servicio postventa, atención al cliente, 
monitoreo de indicadores, seguimiento del cliente, capital relacional, identificación de clientes, definición de funciones y responsabilidades, marketing digital, mantenimiento, control de inventarios, instalaciones adecuadas, equilibrio entre trabajo y vida, plan de carrera, contratación, ambiente laboral, micro-marketing, etc.

Aparte, la literatura identifica un conjunto de capacidades funcionales comunes a las empresas que permiten clasificar las competencias núcleo en cuatro grupos para el funcionamiento organizacional: procesos administrativos, ventas y marketing, procesos y compras y administración del recurso humano. Si bien las MIPES se caracterizan por estructuras formales limitadas, con falta de manuales de procedimientos y de políticas que guíen las acciones internas (Aguirre Choix \& Armenta Velazquez, 2012; Franco-López \& Bedoya-Zapata, 2018), se reconoce la importancia de este tipo de funciones formales que establezcan alternativas de acción a partir de distintas dimensiones como la relación con clientes y proveedores, capacidad de compra, trato al personal, procedimientos y rutinas internas, etc. (Grajales-Gaviria \& Castellanos-Polo, 2018).

En cuanto al primer grupo de capacidades, el proceso administrativo estructura sistemáticamente las funciones de esta naturaleza con el objeto de coordinarlas e integrarlas con el resto de las funciones en cuatro etapas cíclicas (Taylor, 2007): planeación, organización, dirección y control. Las variables a considerar en este estudio son: i) planeación, referido a la toma de decisiones sobre los objetivos empresariales, definición de planes y programas para alcanzarlos; ii) organización, relacionada con el manejo de recursos y actividades para cumplir objetivos y definir puestos, autoridades y responsabilidades; iii) dirección, asociada con la designación de cargos, comunicación, liderazgo y motivación del personal; y, iv) controlar, ligada a la definición de estándares de desempeño y corrección de desviaciones para garantizar se cumpla lo planeado (Chiavenato, 2001).

En el segundo grupo, las ventas se refieren a las actividades necesarias para promover la venta de productos y la interacción entre el personal y con prospectos y clientes; el marketing abarca los programas para persuadir a los clientes potenciales como la publicidad, promoción y distribución. Una combinación de ambas es fuente del crecimiento comercial -atrae nuevos clientes y conserva los existentes- (Rouziés et al., 2005). Siguiendo a Zhang (1999), las variables consideradas se agrupan en promoción, fijación de precios, distribución, servicio al cliente y manejo del producto.

Los procesos y rutinas describen las distintas prácticas y patrones de interacción internos. Se refieren a la manera de hacer las cosas que, al agruparse, conforman las capacidades de la empresa (Teece et al., 1997); son repetitivos y realizados por múltiples actores (Feldman \& Pentland, 2003); detallados, estables, basados en conocimiento interno, replicables, transferibles y con resultados predecibles (Eisenhardt \& Martin, 2000). Asimismo, la capacidad de compra de las empresas es fundamental para la oferta final. El proceso de compra es la función de abastecimiento que impacta en la cantidad, calidad y costos de la oferta (Leenders et al., 1997). Las relaciones cercanas y duraderas con proveedores permiten ventajas en precios y tiempos de entrega. Así, las dimensiones consideradas son, para los procesos, especialización (grado en que los procedimientos se dividen en distintos roles o tareas) y estandarización (grado en que se establecen reglas para procedimientos operativos uniformes) (Cabello Chávez et al., 2004) y, para las compras, en relaciones con proveedores (definición de productos, tiempos, precios y logística) (Zhang, 1999). 
En el último grupo, los recursos humanos se vinculan con el conocimiento, habilidades, experiencias y actitudes incorporados en el personal, por lo que representan competencias que deben ser gestionadas adecuadamente para el éxito de la empresa (Bakator et al., 2018; Chung \& Chen, 2019). Así, las funciones tradicionales de reclutamiento, selección, formación, evaluación de desempeño y compensación del recurso humano son centrales para las empresas de menor tamaño (Mulloli ,2015; Ogunyomi \& Bruning, 2015; Georgiadis \& Pitelis, 2012; Rodríguez, 2012).

También como afirman Briozzo et al., (2017), las empresas con mujeres en puesto de toma de decisiones tienden a mostrar menos niveles de endeudamiento y ventas relativamente altas. Y el género masculino lleva a un ajuste rápido de la MIPE a través de acceso a créditos (Wellalage \& Locke, 2015). Por ende, con el afán de mostrar que la combinación entre género es la clave del éxito empresarial, Hoogendoorn et al., (2013), establecen que empresas con balance entre mujeres y hombres muestran mejores desempeños en términos de ventas y utilidades. Es decir, que no existe diferencia significativa en el desempeño de las empresas diferenciadas por género (Malaya, 2006). El análisis se realiza en torno a las variables desempeño, sistema de recompensas y procesos de información (Zhang, 1999).

Finalmente, la literatura ha estimado un fuerte vínculo entre las dimensiones citadas arriba y el desempeño empresarial. Por ejemplo, Kaynak (2003) y Meunier-FitzHugh y Piercy (2007) encuentran efectos positivos de las prácticas de gestión en indicadores de desempeño como productividad, crecimiento de ventas, utilidades y retornos de la inversión, entre otros. Por su parte, Gilmore (2011) y Mu et al., (2018) indican que una mezcla de marketing interna y externa a la empresa, como fijación de precios, desarrollo de productos, comunicación, etc., constituyen mayores ingresos empresariales y posicionamiento de mercado.

En cuanto a los procesos, Wei et al., (2017) señalan que la flexibilidad en estos, a través de un modelo de negocio, afecta la eficiencia de las empresas. También, Day et al., (2015) sugieren que la capacidad de gestión de los procesos depende de paquetes de rutinas clave consistentes internamente, lo que se asocia con el desempeño operacional (Chin et al., 2012), el rendimiento financiero (Osakwe et al., 2016), el incremento de los ingresos (Peng \& York, 2001) y la reducción de costos de provisión de servicios (Kaleka, 2002). Asimismo, Havlicek (2011) y Ates et al., (2018) indican que la eficiencia en las compras permite costos competitivos y Aboelmaged (2018) concluye que existe una relación positiva entre los procesos manufactureros y las capacidades competitivas de las pequeñas empresas.

Por último, Kesti (2012) sostiene que la calidad de vida laboral, directamente relacionada con la administración eficiente de los recursos humanos y con aspectos como liderazgo, trabajo en equipo y procesos, lleva al desarrollo del personal y a incrementos de la productividad. Como resultado, las competencias núcleo son la razón esencial de la ventaja competitiva de una empresa al crear una diferencia entre el éxito estratégico y el fracaso estratégico (Pehrsson, 2006).

Siguiendo a Prahalad y Hamel (1990), en la medida que las competencias favorezcan el desempeño empresarial tienden a generar una ventaja competitiva. Esta ventaja se alcanza con el uso sistemático de recursos y capacidades clave, que se convierten en core competencies de las empresas (Porter, 1990). Así, siguiendo a Yew Wong y Aspinwall (2005), para ganar ventaja competitiva existen tres estrategias básicas: i) costos, que implica tratar de reducirlos a niveles mínimos en la industria; ii) 
diferenciación, lo que ocurre si el oferente proporciona un producto con valor agregado tal que el mercado lo prefiera; iii) tiempo, referido a que las empresas lideran las tendencias en el mercado o se ajustan rápido a los cambios.

Además, para Alzoubi y Khafajy (2015) el desempeño empresarial sobresaliente exige procesos gerenciales orientados sistemáticamente al cambio. De lo anterior, Sirmon et al., (2007) señalan tres indicadores de desempeño de las empresas: i) rentabilidad, resulta de restar a los ingresos costos e impuestos; ii) crecimiento, es el incremento en ventas e ingresos; iii) sostenibilidad, asociada a la capacidad de la empresa de minimizar efectos negativos de cambios en el contexto global, nacional o local. Estas seis variables se consideran como indicadores del desarrollo empresarial que se generan a partir del cuestionario aplicado.

\section{METODOLOGÍA}

Para identificar las competencias núcleo de las MIPES de Iztapalapa se sigue una metodología de tres pasos. Primero se determinan las capacidades clave. Después se evalúan los rasgos de unicidad, colectividad y flexibilidad estratégica. Para ello, se diseñó, a partir de la revisión bibliográfica especializada, un cuestionario de 45 ítems que permite conocer las competencias empresariales. En términos generales, se estructura en tres grandes secciones. La primera se subdivide en cuatro apartados relativos a un conjunto de capacidades funcionales: procesos gerenciales, ventas y marketing, procesos y compras, y administración del recurso humano, más una sección de identificación de la empresa. Se establecieron preguntas cerradas con respuestas predefinidas con una estructura tipo Likert de cuatro puntos para evitar respuestas neutrales y mejorar la calidad final del cuestionario.

Cada sección consideró una pregunta abierta para conocer la percepción de la relación entre cada dimensión y el desarrollo productivo de la propia empresa. Asimismo, el instrumento consideró una sección con tres preguntas para identificar las core competencies y sus atributos vinculados con la colectividad y la flexibilidad estratégica. La última parte del cuestionario, conformado por seis preguntas, permitió obtener información directamente sobre su desempeño empresarial (en temas de utilidad, diferenciación, costos, ventas, capacidad de ajuste y de cambio).

Se realizó una prueba piloto del cuestionario a un grupo de nueve empresarios MIPES -uno por tamaño y sector- para determinar fortalezas y debilidades del mismo como mecanismo recolector de información. Con esto se realizó la reingeniería del cuestionario, asociada principalmente con los términos empleados. Además, el instrumento se validó por un grupo cinco de expertos en el tema de desarrollo empresarial y se realizaron, por consenso, las modificaciones pertinentes ${ }^{1}$.

Asimismo, se evaluó la consistencia interna, aplicándolo a una muestra de 45 MIPES bajo el criterio de al menos un participante por ítem y que se consideró como un grupo heterogéneo en criterios de edad, género, nivel educativo, giro y años de operación del negocio, etc., para obtener una alta confiabilidad del instrumento. La consistencia interna se determinó mediante el coeficiente Alfa de Cronbach que se basa en el promedio de las correlaciones entre los ítems. Se obtuvo un excelente

\footnotetext{
${ }^{1}$ La versión final del cuestionario está disponible por solicitud a los autores.
} 
coeficiente de 0.94 con $95 \%$ de confianza. Asimismo, no fue necesario eliminar ítems, puesto que hacerlo no condujo a aumentos considerables en la consistencia interna (mayores a 0.01).

El trabajo de campo se realizó en 2017 sobre una muestra representativa de MIPES de Iztapalapa. El universo de MIPES en Iztapalapa en ese año es de 78.746 unidades económicas. Respecto a los perfiles de empresas y personas encuestadas se tiene: i) mayoría del sector servicios (52 \%) frente al industrial (48\%), reflejo del peso de cada uno en la población de unidades económicas en ese territorio; ii) $92 \%$ de las empresas son micro y 8 \% pequeñas, similar a la distribución poblacional de las MIPES; iii) 55 \% de las empresas tiene al menos cinco años de operación, lo que implica una acumulación de conocimientos y experiencias; iv) $54 \%$ de respuestas se obtuvo de mujeres (frente al $46 \%$ sexo masculino); v) $64 \%$ de las respuestas son del dueño y $36 \%$ de algún colaborador, evidenciando la realidad del establecimiento pues son los primeros quienes toman las decisiones.

Se seleccionó una muestra aleatoria, con error del $5 \%$, nivel de confianza del $95 \%$ y probabilidad de ocurrencia entre 5 y $95 \%$, lo que implica 764 unidades empresariales. Al final se obtuvieron 840 cuestionarios válidos, esto es, una muestra total de 840 MIPES. El tamaño muestral obtenido es similar a otros estudios sobre MIPYMES, por ejemplo, Maldonado Guzmán et al., (2010). La muestra alcanzada es representativa en aspectos de pertenencia sectorial, género y años de operación. Cabe señalar que la selección de empresas fue por medio de la técnica no probabilística por conveniencia de juicio del entrevistador, guiado por cuotas de acuerdo con el sector de actividad para reducir el sesgo en las respuestas.

La relación entre las variables identificadas como competencias núcleo y las que miden el desarrollo empresarial se comprueba a través de la estimación de un modelo de corte transversal en donde el desarrollo empresarial (de) es función de un conjunto de competencias núcleo (cn) y una variable de control de género (gen), formalmente:

$$
d e_{i}=\alpha+\beta_{1} c n_{1, i}+\cdots+\beta_{n} c n_{n, i}+\gamma_{1} g e n_{i}+\varepsilon_{i}
$$

Donde de es el desarrollo empresarial aproximado por seis indicadores, que se definen si la empresa: i) se orienta primordialmente a la obtención de utilidad (rentabilidad); ii) registra un aumento en ventas e ingresos (crecimiento); iii) tiene la capacidad de minimizar efectos negativos externos (sostenibilidad); iv) establece una estructura de costos mínimos en la industria (costos); v) lidera las tendencias en el mercado o se ajusta rápido a cambios (tiempo); $y$, vi) ofrece un producto con valor agregado tal que el mercado lo prefiera (diferenciación).

En el cuestionario se obtuvo información explícita que permite operacionalizar estas variables. Así, la rentabilidad se mide por el nivel de utilidad, en pesos corrientes, obtenido por la empresa en el transcurso del último año de operación y hasta el momento de aplicar el instrumento. El crecimiento se definió como el cambio porcentual en el nivel de ventas registrado en ese mismo periodo. La sostenibilidad se aproximó por una variable categórica donde toma el valor de 1 si la empresa enfrentó un cambio negativo en su contexto de negocios y realizó algún ajuste para minimizar dicho efecto, y 0 en caso contrario. Los costos se midieron de dos formas alternativas, primero como porcentaje de las utilidades y como variable dummy con valor de 1 cuando, desde la perspectiva del 
empresario, sus costos están por debajo de los costos de la industria, y 0 en cualquier otro caso. ${ }^{2}$ La variable tiempo se refiere a la flexibilidad de la empresa para ajustarse a cambios oportunamente y/o para introducir estratégicamente los cambios al interior de la empresa. Por tanto, se mide por el número de cambios introducidos en la MIPE (en bienes, servicios, procesos, etc.) en el transcurso del último año. Por último, como proxy de la diferenciación se emplea el número de productos con valor agregado por la propia empresa y que, de acuerdo con el empresario, lo distinga de los productos competidores.

Las variables propuestas para explicar el desarrollo empresarial son el conjunto de competencias núcleo identificadas previamente y que se representan por: i) procesos gerenciales, asociados con las capacidades de planeación, organización, dirección y control; ii) ventas y marketing, vinculado con actividades de promoción de ventas y programas de persuasión de clientes potenciales; iii) procesos y compras, ligado al conjunto de procedimientos, rutinas y métodos para ofrecer bienes y servicios; y, iv) administración del recurso humano, relacionado con la capacidad de coordinar al personal y alcanzar un ambiente laboral adecuado. Las variables anteriores se aproximan, a partir de la información del cuestionario, mediante las respuestas promedio de la empresa en cada rubro. La variable género se incluye para conocer si el género femenino tiene ventaja sobre el masculino, considerando el género reportado del dueño o director de la empresa.

\section{RESULTADOS}

En la aplicación del cuestionario, se solicitó a los colaboradores en las MIPES señalar las principales capacidades funcionales en las cuatro dimensiones generales de la empresa (proceso administrativo, ventas y marketing, procesos y compras y recursos humanos) para evaluarlas en términos de colectividad, unicidad y flexibilidad estratégica. Esto es, además de los ítems propios del cuestionario, los encuestados indicaron dos de sus capacidades empresariales que consideran como una competencia empresarial.

El análisis se realiza para el total de MIPES y para los sectores industriales y de servicios. Los resultados aparecen en la Tabla 1. Como se observa, en promedio para la muestra, las capacidades de ventas y marketing y procesos y compras son las más representativas, las de mayor participación porcentual en el total de respuestas respecto a las capacidades funcionales de la empresa y, por ende, las más viables de significar una competencia central para esas empresas.

Tabla 1. Ranking de capacidades funcionales clave para las competencias, MIPES Iztapalapa Table 1. Ranking of key functional capabilities for the competencies of SMEs in Iztapalapa

\begin{tabular}{lccc}
\multicolumn{1}{c}{ Capacidad } & Industria & Servicios & Total \\
\hline Procesos gerenciales & 6.03 & 15.36 & 10.88 \\
Ventas y marketing & 29.47 & 49.38 & 39.82 \\
Procesos y compras & 57.29 & 16.02 & 35.83 \\
Administración del recurso humano & 7.21 & 19.24 & 13.47 \\
\hline
\end{tabular}

Nota: Participación porcentual de respuestas por capacidad funcional en el total de respuestas. Fuente: elaboración propia.

\footnotetext{
${ }^{2} \mathrm{Al}$ final, la variable proxy empleada es el cociente entre costos y utilidades de la empresa, puesto que la alternativa arrojaba resultados menos significativos.
}

$10 / 25$ 
En el análisis por sector, para la industria los procesos y compras tienen el mayor peso que cualquier otra capacidad. Asimismo, las capacidades de ventas y marketing son relevantes dado que el $29.5 \%$ de las respuestas las señalan como probables de representar una competencia núcleo. Para el sector de servicios, ventas y marketing juegan un papel central en las capacidades con el $49.4 \%$ de las respuestas, seguido por la administración del recurso humano. Desde esta perspectiva, las capacidades de ventas y marketing para las MIPES de servicios y procesos y compras para las industriales significan capacidades clave con potencial de representar competencias núcleo.

Asimismo, los participantes evaluaron la colectividad de las capacidades a partir de los atributos entre-funciones, entre-productos y al-interior-de-la-empresa. Es decir, se les pidió que indicaran en qué medida consideraban a las competencias de su empresa como indispensables para uno o más procesos funcionales, para varios productos y para varias unidades de la empresa, otorgándoles una importancia entre el uno y el cuatro (de menor a mayor magnitud). La Tabla 2 presenta la calificación promedio de los atributos de colectividad para las capacidades clave de la MIPES en la muestra.

Ninguna capacidad es dominante para los tres atributos. No obstante, las capacidades de ventas y marketing registran los mayores promedios para los atributos entre-productos y al-interior-de-laempresa; mientras que las capacidades de administración del recurso humano son primordiales en los atributos entre-funciones y al-interior-de-la-empresa. En concreto, ventas y marketing, además de procesos y compras, son la competencia indispensable para varios productos de las empresas y para varias unidades del negocio. De igual manera, parece que la competencia fundamental para los procesos funcionales de las empresas es la administración del recurso humano. Los procesos gerenciales y el recurso humano son centrales para coordinar funciones entre áreas.

Tabla 2. Colectividad promedio de las competencias en las MIPES de Iztapalapa Table 2. Average collectivity of competencies of SMEs in Iztapalapa

\begin{tabular}{cccc}
\hline Capacidad / Atributo & Entre funciones & Entre productos & Al interior de la empresa \\
\hline & Total & & \\
\hline Procesos gerenciales & 3.11 & 2.51 & 1.89 \\
Ventas y marketing & 2.25 & 3.18 & 3.29 \\
Procesos y compras & 2.14 & 3.15 & 3.14 \\
Administración del recurso humano & 3.20 & 2.67 & 3.29 \\
\hline Procesos gerenciales & Industria & & 1.86 \\
Ventas y marketing & 3.26 & 2.76 & 2.97 \\
Procesos y compras & 1.66 & 2.61 & 3.47 \\
\hline Administración del recurso humano & 2.61 & 3.42 & 3.17 \\
\hline Procesos gerenciales & 2.59 & 2.88 & 1.74 \\
Ventas y marketing & Servicios & & 3.59 \\
Procesos y compras & 2.98 & 2.28 & 2.84 \\
\hline Administración del recurso humano & 2.79 & 3.71 & 3.40 \\
\hline
\end{tabular}

Calificación promedio de los atributos para cada grupo de capacidades.

Fuente: elaboración propia.

Por sector, se observa que las capacidades de procesos gerenciales y de procesos y compras son más relevantes para la industria que para servicios en los tres atributos señalados (Tabla 2). Mediante una 
prueba de hipótesis se determinó que existe diferencia estadísticamente significativa al 95\% de confianza entre las MIPES en la industria y las MIPES en servicios que calificaron a las distintas capacidades como atributos de colectividad apropiados a la empresa. Por ejemplo, para las MIPES que consideraron la capacidad «procesos gerenciales» con una magnitud de 3 y 4 , la $Z$ calculada es 4.022, permitiendo rechazar la hipótesis nula de no diferencia entre muestras en industria y servicios.

Por el contrario, ventas y marketing son más importante para los servicios. Esto está en la línea con la complejidad técnica en la producción industrial y el manejo de bienes y servicios a los clientes o prospectos en la oferta de servicios. Específicamente, procesos y compras son un elemento indispensable para varias unidades, productos y funciones de la empresa, aceptándolos como la capacidad de mayor potencial del sector industrial. Para los servicios, el elemento indispensable son las ventas y marketing como capacidad compartida por productos, funciones y unidades.

Aparte, siguiendo el mismo procedimiento anterior, donde se les pidió a los encuestados en las MIPES calificar en una escala del 1 al 4 respecto a estos atributos, se estableció la unicidad de las competencias núcleo de las MIPES, así como los atributos que la determinan. La Tabla 3 promedia la valoración asignada por los entrevistados. Las capacidades de ventas y marketing muestran los mayores rankings en los tres atributos de unicidad. Esta se distingue, dada la percepción global promedio de 3.11, por la existencia de una experiencia específica en el área (distinto), por el bajo grado en que pueden ser imitados por competidores (no-imitable) y porque tiende a ser irreemplazable por otros activos (no-sustituible). Por ende, ventas y marketing son una competencia central para las MIPES dada su unicidad para competir. Los atributos de unicidad en las restantes capacidades no muestran un patrón dominante.

Asimismo, los procesos y compras son las capacidades con mayor ranking en los tres atributos de unicidad de las MIPES industriales (Tabla 3). Dado que la diferencia con las otras capacidades es estadísticamente significativa (al compararse individualmente con las tres restantes capacidades, se rechaza la hipótesis nula de no diferencias entre muestras con Z mayores a 1.96 en todos los casos), estas capacidades determinan una ventaja competitiva al ser raras, no imitables y no sustituibles. En contraste, la administración del recurso humano es el grupo de capacidades con menor unicidad en los tres atributos. Para las MIPES de servicios la unicidad se basa en ventas y marketing, principalmente. Esto hace que sus ventajas se centren en aspectos de relación con clientes, proveedores y competidores, distribución, promoción y publicad, entre otras, con diferencias estadísticamente significativas al plantear pruebas de hipótesis y calcular Z mayores al de referencia. Los métodos, experiencia y conocimiento (activos intangibles) que emplean configuran una capacidad rara, poco imitable y de baja sustituibilidad.

Finalmente, considerando dos atributos, redespliegue de recursos y reorganización de rutinas, se evalúa la flexibilidad estratégica en las MIPES. Tal como se señaló, la capacidad conjunta para utilizar los activos en aplicaciones para innovar y para reorganizar las rutinas para crear valor significa que la empresa disfruta de una flexibilidad estratégica. El redespliegue de recursos se refiere a la facilidad con la que recursos base de una competencia pueden redesplegarse para desarrollar nuevas capacidades, mientras que la reorganización de rutinas se asocia con la facilidad con la que las rutinas pueden reorganizarse para impulsar el desarrollo empresarial. De nuevo, se les pidió a los colaboradores de las MIPES que calificarán en una escala del 1 al 4 respecto a estos atributos. 
Tabla 3. Capacidades únicas de las MIPES en Iztapalapa

Table 3. Unique capabilities of SMEs in Iztapalapa

\begin{tabular}{|c|c|c|c|}
\hline Capacidad / Atributo & Rareza & No imitable & No Sustituible \\
\hline \multicolumn{4}{|c|}{ Total } \\
\hline Procesos gerenciales & 2.68 & 2.30 & 2.3 \\
\hline Ventas y marketing & 3.11 & 2.83 & 2.64 \\
\hline Procesos y compras & 2.70 & 2.52 & 2.17 \\
\hline Administración del recurso humano & 2.37 & 2.68 & 2.38 \\
\hline \multicolumn{4}{|c|}{ Industria } \\
\hline Procesos gerenciales & 2.63 & 2.56 & 2.55 \\
\hline Ventas y marketing & 3.03 & 2.76 & 2.41 \\
\hline Procesos y compras & 3.12 & 2.96 & 2.59 \\
\hline Administración del recurso humano & 2.29 & 2.39 & 1.98 \\
\hline \multicolumn{4}{|c|}{ Servicios } \\
\hline Procesos gerenciales & 2.71 & 2.12 & 2.11 \\
\hline Ventas y marketing & 3.17 & 2.88 & 2.80 \\
\hline Procesos y compras & 2.41 & 2.20 & 1.97 \\
\hline Administración del recurso humano & 2.43 & 2.88 & 2.66 \\
\hline
\end{tabular}

Calificación promedio de los atributos para cada grupo de capacidades.

Fuente: elaboración propia.

En la Tabla 4, se sintetizan las respuestas promedio. Las ventas y marketing, seguidas por procesos y compras, tienen la mayor flexibilidad respecto a la utilización de recursos como vía para crear capacidades en la empresa. Esto implica que entre más empleen estos recursos en actividades operativas y estratégicas, mayores capacidades de competir desarrollan. Los procesos gerenciales muestran el menor potencial para crear nuevas capacidades, que se explica por la baja profesionalización y educación de dueños/directivos de este tipo de empresas. Ventas y marketing y procesos y compras tiene el mayor potencial en la reorganización de rutinas, confirmándose su rol como competencias centrales.

Sectorialmente se identifica que, para las MIPES de industria, procesos y compras y ventas y marketing son las de mayor flexibilidad por la posibilidad de aplicarse en el impulso de nuevas capacidades y reorganizarse para favorecer el desempeño futuro (Tabla 4). Para las MIPES de servicios ventas y marketing y administración del recurso humano tienen el mayor potencial para generar nuevas capacidades que las doten de ventaja competitiva; mientras que administración del recurso humano y ventas y marketing tienen potencial de reorganización para favorecer su desarrollo.

En consecuencia, se establece a las capacidades de ventas y marketing y de procesos y compras como competencias núcleo para este grupo de empresas. Asimismo, la relevancia de ventas y marketing para servicios, y procesos y compras para industria, se confirman desde un enfoque estratégico, entendiéndolas como las competencias necesarias para la generación de nuevas capacidades y el impulso futuro de la empresa. 
Tabla 4. Flexibilidad estratégica de las capacidades en las MIPES de Iztapalapa Table 4. Strategic flexibility of the capabilities of SMEs in Iztapalapa

\begin{tabular}{|c|c|c|}
\hline Capacidad / Atributo & Redespliegue de recursos & Reorganización de rutinas \\
\hline \multicolumn{3}{|c|}{ Total } \\
\hline Procesos gerenciales & 2.52 & 2.67 \\
\hline Ventas y marketing & 2.96 & 2.92 \\
\hline Procesos y Compras & 2.88 & 2.78 \\
\hline Administración del Recurso Humano & 2.62 & 2.66 \\
\hline \multicolumn{3}{|c|}{ Industria } \\
\hline Procesos gerenciales & 2.49 & 2.37 \\
\hline Ventas y marketing & 2.92 & 2.91 \\
\hline Procesos y compras & 3.19 & 3.02 \\
\hline Administración del recurso humano & 2.39 & 2.37 \\
\hline \multicolumn{3}{|c|}{ Servicios } \\
\hline Procesos gerenciales & 2.55 & 2.92 \\
\hline Ventas y marketing & 2.99 & 2.91 \\
\hline Procesos y compras & 2.60 & 2.55 \\
\hline Administración del recurso humano & 2.83 & 2.93 \\
\hline
\end{tabular}

Como se estableció, las MIPES alcanzan una ventaja competitiva cuando las competencias núcleo impulsan su desempeño empresarial. La contribución de las competencias núcleo de las MIPES de Iztapalapa en el desarrollo empresarial se estima econométricamente siguiendo una metodología de corte transversal (información para un solo año y 840 unidades empresariales), que permite examinar las variaciones en la información a través de las micro y pequeñas empresas a partir de una función tipo Cobb-Douglas. Como los resultados derivados de un análisis de corte transversal en muestras heterogéneas deben tomarse con cautela, el análisis se realiza para el total de la muestra y para los sectores industrial y de servicios, donde el grado de homogeneidad es mayor y se controla mediante la variable género.

La validez de las estimaciones econométricas se verifica a través de pruebas de hipótesis estructurales convencionales. Así, las matrices de correlación señalan coeficientes, si bien relativamente bajos, la mayoría de ellos con niveles de significancia del 0.01 y del 0.10 significativos, sugiriendo la existencia de una asociación lineal entre las competencias núcleo (variables explicativas) y las asociadas al desempeño empresarial (variables dependientes). Como la raíz cuadrada del coeficiente de determinación del modelo (R2) estimado por Mínimos Cuadrados Ordinarios (MCO) es más grande que el coeficiente de correlación entre regresores, para todas las estimaciones, se acepta la existencia de no multicolinealidad. De igual modo, se prueba que los componentes del vector de errores tengan igual varianza, problema común en los modelos de corte transversal, dada la heterogeneidad de las observaciones. Acá, el test Breusch-Pagan no rechaza la hipótesis de homocedasticidad (el estadístico X2 varía en un rango entre 265.98 y 292.79 para las distintas regresiones).

Aunque no es crucial para muestras grandes como en este caso, se prueba la distribución de los errores. El estadístico Jarque-Bera, que se distribuye asintóticamente como $\chi 2$, es para todos los casos menores a 0.0278 , por lo que los errores del modelo se distribuyen aproximadamente 
normales. Por tanto, se acepta que las estimaciones son válidas y aportan información del efecto de las competencias centrales sobre distintas medidas de desempeño de las MIPES.

Los resultados se muestran en la Tabla 5. En general, los resultados obtenidos fueron los esperados, esto es, las competencias núcleo impactan positiva y significativamente en el desarrollo de las empresas locales prácticamente desde cualquiera de los indicadores, comprobándose así la hipótesis planteada.

En general, independientemente del sector empresarial, se estima un efecto positivo y significativo de al menos dos tipos de grupos de capacidades en la variable dependiente. Las capacidades asociadas con los procesos gerenciales en las MIPES industriales afectan de manera significativa a todas las variables de desempeño empresarial, excepto el crecimiento de las ventas. Al mismo tiempo, las capacidades relacionadas con ventas y marketing en las empresas de servicios explican todas las medidas de desempeño de las MIPES.

En concreto, respecto a la contribución de las competencias identificadas como centrales para las MIPES en el desempeño empresarial, se observa que estas competencias en cada sector son, en promedio para todos los indicadores de desarrollo, las de mayor efecto. Esto es, para la industria, procesos y compras que tienen un efecto medio en el desempeño productivo de 0.254 , seguido de la variable procesos gerenciales (0.252). Para los servicios las competencias de ventas y marketing tiene, en promedio, el coeficiente más alto, seguido por las de administración del recurso humano.

Tabla 5. Efecto de las Competencias Núcleo en el desarrollo empresarial de MIPES de Iztapalapa, por sector Table 5. Effect of core competencies on the business development of SMEs in Iztapalapa by sector

\begin{tabular}{|c|c|c|c|c|c|c|}
\hline \multicolumn{7}{|c|}{ Elasticidades Cobb-Douglas } \\
\hline Variables & Costos & Diferenciación & Tiempo & Rentabilidad & Crecimiento & Sostenibilidad \\
\hline \multicolumn{7}{|c|}{ Sector Industrial } \\
\hline C & 1.667 & 3.36 & 4.087 & 3.048 & 2.829 & 2.281 \\
\hline Procesos gerenciales & $0.239^{* *}$ & $0.219^{* *}$ & $0.377^{* *}$ & $0.170^{* *}$ & 0.259 & $0.108^{* *}$ \\
\hline Ventas y marketing & -0.169 & $0.191^{* *}$ & $0.131^{* *}$ & $0.140^{*}$ & $0.184^{*}$ & -0.299 \\
\hline Procesos y compras & $0.324^{*}$ & 0.196 & $0.277^{* *}$ & -0.099 & $0.162^{*}$ & 0.193 \\
\hline Administración del recurso humano & -0.156 & 0.141 & 0.132 & $0.219^{* * *}$ & 0.29 & $0.167^{* *}$ \\
\hline Género & 0.081 & -0.101 & $-0.208^{* *}$ & 0.108 & -0.175 & $0.319^{* *}$ \\
\hline $\mathrm{R}^{2}$ & 0.71 & 0.70 & 0.75 & 0.72 & 0.65 & 0.66 \\
\hline $\mathrm{N}$ & 403 & 403 & 403 & 403 & 403 & 403 \\
\hline \multicolumn{7}{|c|}{ Sector Servicios } \\
\hline C & 1.947 & 2.974 & 2.995 & 3.587 & 1.916 & 2.181 \\
\hline Procesos gerenciales & -0.309 & $0.292^{*}$ & $0.137^{* *}$ & 0.262 & 0.256 & $0.216^{*}$ \\
\hline Ventas y marketing & $-0.178^{* *}$ & $0.272^{*}$ & $0.301^{*}$ & $0.164^{* *}$ & $0.302^{*}$ & $0.405^{*}$ \\
\hline Procesos y compras & $0.079^{* *}$ & 0.172 & 0.311 & 0.324 & -0.304 & -0.141 \\
\hline Administración del recurso humano & 0.319 & -0.132 & $0.257^{*}$ & $0.099^{*}$ & $0.212^{* *}$ & $0.266^{* *}$ \\
\hline Género & 0.516 & 0.276 & $0.116^{*}$ & 0.206 & $0.074^{* * *}$ & 0.324 \\
\hline $\mathrm{R}^{2}$ & 0.72 & 0.73 & 0.76 & 0.70 & 0.71 & 0.67 \\
\hline $\mathrm{N}$ & 437 & 437 & 437 & 437 & 437 & 437 \\
\hline
\end{tabular}

* Nivel de significancia de 0.01; ** nivel de significancia de 0.05; *** nivel de significancia de 0.10. Fuente: elaboración propia. 


\section{DISCUSIÓN}

Para el caso del sector industrial las competencias de procesos y compras contribuyen a que las MIPES mantengan costos bajos, a un rápido ajuste ante cambios en el mercado y a un incremento permanente en ventas e ingresos. Esto significa que los procesos implementados son relativamente eficientes y lo suficientemente flexibles para adecuarse a las necesidades del mercado. En línea con este resultado, Aboelmaged (2018) afirma que existe una relación positiva entre procesos manufactureros y capacidades competitivas de las pequeñas empresas. La mayor eficiencia en estos procesos, representaría profundizar la capacidad como competencia central, al tiempo que favorece el desempeño de las MIPES en este sector.

También, como se dijo, los procesos gerenciales, entendidos como procesos de toma de decisiones, mantiene una contribución alta en el desarrollo de la empresa, afectando positivamente prácticamente a todos los indicadores. En concreto, esta variable afecta notablemente el tiempo de respuesta de la empresa a los nuevos requerimientos impuestos desde el entorno; por otro lado, estas capacidades son clave para establecer procesos de cambio e innovación en la empresa. Para Chin et al., (2012) los procesos gerenciales y el tamaño de las empresas contribuyen a la flexibilidad en la realización de sus tareas. En particular, para Alzoubi y Khafajy (2015), el desempeño empresarial sobresaliente exige procesos gerenciales orientados sistemáticamente al cambio.

Desde el enfoque de género, el femenino tiene una contribución superior en la capacidad de la empresa de minimizar los efectos negativos de los cambios en el contexto (Rodríguez-Jiménez et al., 2017). Briozzo et al., (2017) señalan que las empresas con mujeres en puestos de toma de decisiones tienden a mostrar menores niveles de endeudamiento y ventas relativamente altas. No obstante, el género masculino lleva a un ajuste más rápido de las MIPES, por ejemplo, a través del acceso a créditos (Wellalage \& Locke, 2015). Estos resultados opuestos se reflejan en las estimaciones, puesto que la superioridad del género femenino en las MIPES solo se presenta en la variable tiempo para ambos sectores, en la sostenibilidad para la industria y para el crecimiento en servicios. Por ende, la evidencia para asociarse más con los resultados de Hoogendoorn et al., (2013), quienes establecen que empresas con balance entre mujeres y hombres muestran mejores desempeños en términos de ventas y utilidades.

Por otro lado, para las MIPES en servicios las competencias de ventas y marketing contribuyen a prácticamente todas las medidas empresariales. Esto se alinea a los resultados de Meunier-FitzHugh y Piercy (2007) para quienes la relación entre ambas capacidades conduce a mejores resultados empresariales. Estas capacidades clave llevan a reducir los efectos negativos derivados de cambios externos y a una pronta respuesta a través de métodos de marketing que modifican sus servicios (Gilmore, 2011), así como al incremento de ventas e ingresos que se refleja en utilidades. Al respecto, Osakwe et al., (2016) argumentan sobre la necesidad de desarrollar capacidades empresariales clave, particularmente orientación a la marca y sensibilidad al mercado, para que este grupo de empresas sea financieramente viable. No obstante, se estima un signo negativo respecto a los costos. Esto puede implicar que la estrategia se oriente hacia esfuerzos de ventas y marketing sin que sea costoeficientes, por lo que el costo total de la empresa puede incrementarse limitando su competitividad. Una idea similar se presenta en Havlicek (2011). 
Asimismo, una fuerza competitiva es la administración del recurso humano al contribuir a las variables tiempo, rentabilidad, crecimiento y sostenibilidad. Al respecto, Bakator et al., (2018) concluyen que existe una relación positiva entre prácticas gerenciales del recurso humano y el desempeño global de la empresa, a través de la mejora en el bienestar y productividad de los trabajadores, que se traduce en un clima organizacional adecuado para el desempeño de la empresa.

Para el caso de las MIPES, esto puede entenderse dado el relativo bajo número de colaboradores y el tipo de bien intangible que ofrecen, haciendo que las relaciones laborales adquieran mayor peso. Así, el ajuste (y rapidez) para minimizar efectos negativos y las ventas, ingresos y utilidad se explican por aspectos ligados al ambiente laboral, resultados análogos a los de Mulloli (2015), Ogunyomi y Bruning (2015) y Georgiadis y Pitelis (2012) quienes señalan que políticas de compensación, recompensa y desarrollo del personal conducen al crecimiento de las empresas y a un mejor desempeño financiero.

Las MIPES con abundancia relativa del género femenino muestran una ventaja competitiva, puesto que conducen a un ajuste más rápido ante cambios en las condiciones del mercado o a introducir primero elementos vanguardistas. Simultáneamente, su contribución en los procesos de ventas para generar ingresos es mayor en comparación con la de los hombres, lo que contrasta, sin embargo, con la literatura; por ejemplo, Malaya (2006) no encuentra diferencia significativa en el desempeño de las empresas diferenciadas por género.

Por último, se observa que una estructura de bajos costos se alcanza más por las empresas industriales, mientras que la reducción del impacto de los cambios externos se presenta más por las capacidades de las empresas de servicios. En consecuencia, las ventajas competitivas del sector industrial, basadas en competencias núcleo de procesos y compras, llevan a que se especialicen en contención de costos siguiendo estrategias basadas en procesos internos relativamente complejos. En otras palabras, cabe esperar que sus sistemas de costos se basen en órdenes de producción y que sus estructuras de costos estén ligadas principalmente a la materia prima y mano de obra, como señala Gómez Niño (2011).

Las ventajas del sector servicios, a partir de competencias de ventas y marketing, conducen a especializarse en la minimización de efectos negativos y a enfocarse en resultados, al emplear procesos cercanos al cliente final y con productos ligeramente diferenciados, lo que se asocia con una estrategia de orientada al servicio que se ha identificado por Benyoussef Zghidi y Zaiem (2017) como clave para el positivo desempeño empresarial.

\section{CONCLUSIONES}

Las competencias núcleo de las micro y pequeñas empresas pueden desarrollarse para mejorar los estándares de desempeño y, al ser base de la producción local, propiciar el crecimiento del territorio. Al respecto, para que una empresa alcance una competencia central, debe contar con recursos (tangibles e intangibles) únicos, que le permitan explotarla eficientemente de forma diferenciada a los competidores. 
Los resultados evidencian que el desarrollo de la muestra de MIPES en Iztapalapa depende positivamente de las competencias núcleo que, a su vez, son función de capacidades clave, aceptándose la hipótesis planteada. En este sentido, se consideraron cuatro grupos de capacidades principales de las MIPES: procesos gerenciales, ventas y marketing, procesos y compras y administración del recurso humano, lo que permitió establecer que para ambos sectores las capacidades clave se caracterizan por ser raras, no imitables y no sustituibles. De igual manera, se definió que las capacidades clave son estratégicas para el redespliegue de recursos y para la reorganización de rutinas.

Se concluye que las competencias núcleo impactan positiva y significativamente, desde casi todos los indicadores, en el desarrollo empresarial cumpliendo con la hipótesis planteada. Para las MIPES industriales las competencias de procesos y compras contribuyen a mantener costos bajos, a un rápido ajuste ante cambios en el mercado y a un incremento permanente en ventas e ingresos. Para las MIPES de servicios las competencias de ventas y marketing favorecen la reducción de efectos negativos por cambios en el ambiente global o local, implicando una pronta respuesta y un incremento de ventas e ingresos reflejado en utilidades.

En concreto, se tiene que las MIPES en ambos sectores siguen estrategias de carácter defensivo para proteger su mercado ante amenazas del mercado o la industria. Por ende, se requieren políticas de fomento empresarial con carácter sectorial a través del impulso de competencias núcleo. Es necesario el diseño de programas de desarrollo de procesos de producción para empresas industriales y de competencias en ventas y marketing para las de servicios. El efecto esperado es que las primeras configuren estructuras de costos por debajo del promedio de la industria y que las segundas minimicen los efectos negativos de competidores de mayor tamaño.

\section{CONFLICTOS DE INTERÉS}

Los autores declaran que no presentan conflictos de interés financiero, profesional o personal que pueda influir de forma inapropiada en los resultados obtenidos o las interpretaciones propuestas.

\section{CONTRIBUCIÓN DE AUTORES}

Para el desarrollo de este proyecto todos los autores han realizado una contribución significativa especificada a continuación:

Omar Neme-Castillo: desarrolló la metodología propuesta para la investigación. Realizó las gráficas de datos y resultados. Revisó y corrigió el documento.

Ana Lilia Valderrama-Santibáñez: desarrolló la introducción y la conclusión del documento y discutió los resultados. Revisó y corrigió el documento.

Cesaire Chiatchoua: desarrolló la temática en relación a las Competencias núcleo. Colaboró en el desarrollo de la metodología. Revisó y corrigió el documento. 


\section{REFERENCIAS}

Aboelmaged, M. (2018). The drivers of sustainable manufacturing practices in Egyptian SMEs and their impact on competitive capabilities: A PLS-SEM model. Journal of Cleaner Production, v. 175, 207-221. https://doi.org/10.1016/j.jclepro.2017.12.053

Aguirre Choix, R.; Armenta Velazquez. C. E. (2012). La importancia del control interno en las pequeñas y medianas empresas en México. El Buzón de Pacioli, n. 76, 1-17. URL

Ali, M. S. Y. (2017). Moderating effect of support service quality on marketing IS sophistication and bank's core competencies. Journal of Islamic Marketing, v. 8, n. 4, 711-731. https://doi.org/10.1108/JIMA-10-2015-0078

Álvarez, M.; Durán, J. (2009). Manual de la Micro, Pequeña y Mediana Empresa: una contribución a la mejora de los sistemas de información y el desarrollo de las políticas públicas. German Agency for Technical Cooperation, Centro Regional de Promoción de la MIPYME. URL

Alzoubi, H. M.; Khafajy, N. A. (2015). The impact of business process performance on business performance superiority. International Journal of Business and Management Review, v. 3, n. 2, 17-34. URL

Andriessen, D.; Frijlink Ra, M.; Van Gisbergen, I.; Blom, J. (1999). A core competency approach to valuing intangible assets. International Symposium Measuring and Reporting Intellectual Capital: Experiences, Issues, and Prospects, Amsterdam. URL

Ates, M. A.; van Raaij, E. M; Wynstra, F. (2018). The impact of purchasing strategy-structure (mis)fit on purchasing cost and innovation performance. Journal of Purchasing and Supply Management, v. 24, n. 1, 68-82. https://doi.org/10.1016/i.pursup.2017.05.002

Bakator, M.; Petrovic, N.; Boric, S.; Dalic, N. (2018). Uticaj upravljanja ljudskim resursima na poslovne performance - sistematski pregled literature. Journal of Engineering Management and Competitiveness, v. 9, n. 1, 3-13. https://doi.org/10.5937/JEMC1901003B

Bakker, H.; Wynford, J.; Michele, N. (1994). Using core competencies to develop new business. Long Range Planning, v. 27, n. 6, 13-27. https://doi.org/10.1016/0024-6301(94)90161-9

Barney, J. (1991). Firm resources and sustained competitive advantage. Journal of Management, v. 17, n. 1, 99-120. https://doi.org/10.1177\%2F014920639101700108

Benyoussef Zghidi, A.; Zaiem, I. (2017). Service orientation as a strategic marketing tool: the moderating effect of business sector. Competitiveness Review, v. 27, n. 1, 40-61. https://doi.org/10.1108/CR-02-2015-0012

Briozzo, A.; Albanese, D.; Santolíquido, D. (2017). Corporate governane, financing and gender: a study of SMESs from Argentinean security markets. Contaduría y Administración, v. 62, n. 2 358-376. https://doi.org/10.1016/i.cya.2017.03.002 
Cabello Chávez, A.; Reyes Avellaneda, R.; Solís Pérez, P. C. (2004). El perfil organizacional de las PyMEs (Microempresas, pequeñas y medianas empresas) en el sector manufacturero: un análisis integral. Congreso Anual Internacional. Academia de Ciencias Administrativas, Acapulco. URL

Cantú Mata, J. L.; Palomo González, M. A.; Segoviano Hernández, J.; González Duéñez, V. P. (2012). Variables que influyen en el desarrollo de las competencias centrales, al requerir software en la empresa. Innovaciones de Negocios, v. 9, n. 17, 41-55. URL

Castro, M. E. (2010). Las estrategias competitivas y su importancia en la buena gestión de las empresas. Ciencias Económicas, v. 28, n. 1, 247-276. URL

Chen, C.; He, X.; Liu, Z.; Sun, W.; Dong, H.; Chu, Y. (2020). Analysis of regional economic development based on land use and land cover change information derived from Landsat imagery. Scientific Reports, v. 10, n. 12721. https://doi.org/10.1038/s41598-020-69716-2

Chiavenato, I. (2001). Administración: teoría, proceso y práctica. McGraw-Hill.

Chin, T. A.; Hamid, A. B. A.; Rasli, A.; Baharun, R. (2012). Adoption of supply chain management in SMEs. Procedia Social and Behavioral Sciences, v. 65, 614-619. https://doi.org/10.1016/i.sbspro.2012.11.173

Chung, C. H.; Chen, L. J. (2019). Text mining for human resources competencies: Taiwan example. European Journal of Training and Development, v. ahead-of-print, n. ahead-of-print. https://doi.org/10.1108/EJTD-07-2018-0060

Clardy, A. (2007). Strategy, core competencies and human resource development. Human Resource Development International, v. 10, n. 3, 339-349. https://doi.org/10.1080/13678860701516628

Day, M.; Lichtenstein, S.; Samouel, P. (2015). Supply management capabilities, routine bundles and their impact on firm performance. International Journal of Production Economics, v. 164, 1-13. https://doi.org/10.1016/i.ijpe.2015.02.023

Díaz, A. (2003). Efectos de la globalización en la competitividad y en los sistemas productivos locales en México. Observatorio de la Economía Latinoamericana, n. 5. URL

Dini, M.; Stumpo, G. (2018). MIPYMES en América Latina: un frágil desempeño y nuevos desafíos para las políticas de fomento. Comisión Económica para América Latina y el Caribe CEPAL.

Eisenhardt, K. M.; Martin, J. A. (2000). Dynamic capabilities: what are they? Strategic Management Journal, v. 21, n. 10-11, 1105-1121. https://doi.org/10.1002/1097-0266(200010/11)21:10/11\%3C1105::AID-SMJ133 \%3E3.0.CO;2$\underline{E}$

Feldman, B.; Pentland, B. (2003). Reconceptualizing organizational routines as a source of flexibility and change. Administrative Science Quarterly, v. 48, n. 1, 94-118.

https://doi.org/10.2307/3556620 
Francioli, F.; Albanese, M. (2017). The evaluation of core competencies in networks: the network competence report. Journal of Intellectual Capital, v. 18, n. 1, 189-216. https://doi.org/10.1108/JIC-01-2016-0014

Franco-López, J. A.; Bedoya-Zapata, J. (2018). Análisis de modelos del talento humano aplicados en organizaciones pymes. Revista CEA, v. 4, n. 7, 85-101. https://doi.org/10.22430/24223182.761

Georgiadis, A.; Pitelis, C. N. (2012). Human resources and SME performance in services: Empirical evidence from the UK. The International Journal of Human Resource Management, v. 23, n. 4, 808-825. https://doi.org/10.1080/09585192.2011.561236

Gilmore, A. (2011). Entrepreneurial and SME marketing. Journal of Research in Marketing and Entrepreneurship, v. 13, n. 2, 137-145. https://doi.org/10.1108/14715201111176426

Gómez Niño, O. (2011). Los costos y procesos de producción, opción estratégica de productividad y competitividad en la industria de confecciones infantiles de Bucaramanga. Revista Escuela de Administración de Negocios, n. 70, 167-180. URL

Goold, M.; Campbell, A.; Marcus, A. (1997). Leveraging competencies across business. In Campbell, A.; Sommers Luchs, K. (Ed.). Core competency-based strategy (295-320). International Thomson Business Press.

Grajales-Gaviria, D. A.; Castellanos-Polo, O. C. (2018). Evaluación del control interno en el proceso de tesorería de las pequeñas y medianas empresas de Medellín. Revista CEA, v. 4, n. 7, 67-83. https://doi.org/10.22430/24223182.760

Grant, R. (1991). The resource-based theory of competitive advantage: implications for strategy formulation. In Zack M. H. (Ed.), Knowledge and Strategy. Elsevier Inc. URL

Hafeez, K.; Zhang, Y.; Malak, N. (2002). Core competence for sustainable competitive advantage: A structured methodology for identifying core competence. IEEE Transactions on Engineering Management, v. 49, n. 1, 28-35. https://doi.org/10.1109/17.985745

Havlicek, K. (2011). Marketing management and marketing controlling of SMEs. European Research Studies Journal, v. 14, n. 4, 39-54. URL

Helleloid, D.; Simonin, B. (1994). Organisational learning and a firm's core competence. En Hamel, G.; Heene, A. (Ed.). Competence-based competition (213-239). John Wiley \& Sons.

Herhausen, D.; Morgan, R. E.; Brozović, D.; Volberda, H. W. (2020). Re-examining strategic flexibility: a meta-analysis of its antecedents, consequences and contingencies. British Journal of Management, v. 00, 1-21. https://doi.org/10.1111/1467-8551.12413 
Hoogendoorn, S.; Oosterbeek, H.; van Praag, M. (2013). The impact of gender diversity on the performance of business teams: evidence from a field experiment. Management Science, v. 59, n. 7, 1479-1724. https://doi.org/10.1287/mnsc.1120.1674

Huang, H.; He, Y.; Chen, J. (2019). Competitive strategies and quality to counter parallel importation in global market. Omega, v. 86, 173-197. https://doi.org/10.1016/j.omega.2018.07.009

Instituto Nacional de Estadística y Geografía (2016). Se difunden estadísticas detalladas sobre las micro, pequeñas y medianas empresas del país, Boletín de Prensa, n. 285/16.

Instituto Nacional de Estadística y Geografía. (2017). Anuario estadístico y geográfico de la Ciudad de México 2017. Instituto Nacional de Estadística y Geografía.

Instituto Nacional de Estadística y Geografía (2019). INEGI presenta resultados de la encuesta nacional sobre productividad y competitividad de las micro, pequeñas y medianas empresas (ENAPROCE) 2018. Comunicado de prensa, n. 448/19.

Kaleka, A. (2002). Resources and capabilities driving competitive advantage in export markets: guidelines for industrial exporters. Industrial Marketing Management, v. 31, n. 3, 273-283. https://doi.org/10.1016/S0019-8501(00)00148-6

Kaynak, H. (2003). The relationship between total quality management practices and their effects on firm performance. Journal of Operations Management, v. 21, n. 4, 405-435. https://doi.org/10.1016/S0272-6963(03)00004-4

Kesti, M. (2012). Organization human resources development connection to business performance. Procedia Economics and Finance, v. 2, 257-264. https://doi.org/10.1016/S2212-5671(12)00086-X

Leenders, M. R.; Fearon, H. E.; England, W. B. (1997). Administración de compras y materiales. CECSA.

Malaya, M. F. (2006). A gender-based analysis of performance of small and medium printing firms in Metro Manila. Journal of International Women's Studies, v. 8, n. 1, 83-95. URL

Maldonado Guzmán, G.; Martínez Serna, M. C.; García Perez de Lema, D.; Aguilera Enríquez, L.; González Adame, M. (2010). La influencia de las TICs en el rendimiento de la PyME de Aguascalientes. Investigación y Ciencia, v. 18, n. 47, 57-65. URL

Meunier-FitzHugh, K. L.; Piercy, N. F. (2007). Does collaboration between sales and marketing affect business performance? The Journal of Personal Selling \& Sales Management, v. 27, n. 3, 207220. https://doi.org/10.2753/PSS0885-3134270301

Moingeon, B.; Ramanantsoa, B.; Metais, E.; Orton, J. D. (1998). Another look at strategy-structure relationships: the resource-based view. European Management Journal, v. 16, n. 3, 297-305. https://doi.org/10.1016/S0263-2373(98)00006-1 
Molina, R. (2009). La incidencia de las competencias esenciales y el propietario-dirigente en el fuerte crecimiento de la pyme manufacturera en San Luis Potosí. Cuadernos de Administración, v. 22, n. $38,121-144 . \underline{U R L}$

Molina Sánchez, R.; López Salazar, A.; Contreras Soto, R. (2013). El emprendimiento y crecimiento de las PYMES. Acta Universitaria, v. 24, n. 1, 59-72. URL

Mu, J.; Baob, Y.; Sekhon, T., Qi, J.; Love, E. (2018). Outside-in marketing capability and firm performance. Industrial Marketing Management, v. 75, 37-54. https://doi.org/10.1016/j.indmarman.2018.03.010

Mulloli, E.; Islami. X.; Skenderi, N. (2015). Human resource management practices and SMEs performance: study based in Kosovo. International Review of Management and Business Research, v. 4, n. 4, 1171-1179. URL

Ogunyomi, P.; Bruning, N. S. (2015). Human resource management and organizational performance of small and medium enterprises (SMEs) in Nigeria. The International Journal of Human Resource Management, v. 27, n. 6, 612-634. https://doi.org/10.1080/09585192.2015.1033640

Osakwe, C. N.; Chovancovan, M.; Ogbonna, B. U. (2016). Linking SMEs profitabilityt to brand orientation and market-sensing capability: a service sector evidence. Periodica Polytechnica Social and Management Sciences, v. 24, n. 1, 34-40. https://doi.org/10.3311/PPso.8069

Pehrsson, A. (2006). Business relatedness measurements: state-of-the-art and a proposal. European Business Review, v. 18, n. 5, 350-363. https://doi.org/10.1108/09555340610686949

Peng, M. W.; York, A. S. (2001). Behind intermediary performance in export trade: transactions, agents and resources. Journal of International Business Studies, v. 32, 327-346. https://doi.org/10.1057/palgrave.jibs.8490955

Porter, M. E. (1990). The competitive advantage of nations. Free Press.

Prahalad, C. K.; Hamel, G. (1990). The core competence of the corporation. En Hahn D., Taylor B. (Ed.). Strategische Unternehmungsplanung - Strategische Unternehmungsführung (275-292). Berlin, Heidelberg: Springer. https://doi.org/10.1007/3-540-30763-X 14

Prahalad, C. K.; Hamel, G. (1994). Competing for the future. Harvard Business School Press.

Reed, R.; De Fillippi, R. J. (1990). Causal ambiguity, barriers to imitation, and sustainable competitive advantage. Academy of Management Review, v. 15, n. 1, 88-102. https://doi.org/10.5465/amr.1990.4308277

Rodríguez, D. (2012). Prácticas de gestión humana en pequeñas empresas. Apuntes del CENES, v. 31, n. 54, 193-226. URL 
Rodríguez-Jiménez, M.; Leiva, J. C.; Castrejón-Mata, C. (2017). ¿Cómo son las mujeres que lideran PYMES? Un estudio aplicado en Costa Rica. Revista CEA, v. 3, n. 5, 29-40. https://doi.org/10.22430/24223182.646

Rouziés, D.; Anderson, E.; Kohli, A. K.; Michaels, R. E.; Weitz, B. A.; Zolterns, A. A. (2005). Sales and marketing integration: A proposed framework. Journal of Personal Selling \& Sales Management, v. 25, n. $2,113-122$. URL

Sadler, P. (2003). Strategic Management (2da Ed.). Kogan Page.

Sánchez, R. (1995). Strategic flexibility in product competition. Strategic Management Journal, v. 16, n. 1 S, 135-159. https://doi.org/10.1002/smj.4250160921

Sánchez Aguirre, D. P.; Maldonado Alcudia, M. C.; Martínez Vásquez, L. D.; Lara, G. (2017). Rentabilidad de hoteles boutique explicada desde la experiencia memorable. El Periplo Sustentable, n. 33, 81-104. URL

Senthilkumar, S.; Marutha Durai, M.; Sharmila, A.; Poornima, J. (2013). Business Policy and Strategic Management. S. Chand \& Company PVT.

Sirmon, D. G., Hitt, M. A., \& Ireland, R. D. (2007). Managing firm resources in dynamic environments to create value: looking inside the black box. Academy of Management Review, v. 32, n. 1, 273292. https://doi.org/10.5465/amr.2007.23466005

Taylor, F. W. (2007). The principles of scientific management. McGraw-Hill.

Teece, D. J.; Pisano, G.; Shuen, A. (1997). Dynamic capabilities and strategic management. Strategic Management Journal, v. 18, n. 7, 509-533. https://doi.org/10.1002/(SICI)1097-0266(199708)18:7\%3C509::AID-SMJ882 \%3E3.0.CO;2-Z

Universidad Nacional Autónoma de México. (2011). Propuesta para el desarrollo económico, social y urbano en el Distrito Federal. Universidad Nacional Autónoma de México. URL

Volberda, H. W. (1996). Flexible configuration strategies within Philips semiconductors: A strategic process of entrepreneurial revitalisation. In Sánchez, R.; Heene, A.; Thomas, H. (Ed.). Dynamics of competence-based competition: Theory and practice in the new strategic management (229278). Elsevier.

Wei, Z.; Song, X.; Wang, D. (2017). Manufacturing flexibility, business model design, and firm performance. International Journal of Production Economics, v. 193, 87-97. https://doi.org/10.1016/j.ijpe.2017.07.004

Wellalage, N.; Locke, S. (2015). Access to credit by SMEs in South Asia: do women entrepreneurs face discrimination. Research in International Business and Finance, v. 41, 336-346. https://doi.org/10.1016/j.ribaf.2017.04.053 
Williams, J. R. (1992). How sustainable is your competitive advantage? California Management Review, v. 34, n. 3, 29-51. https://doi.org/10.2307\%2F41167422

Yew Wong, K.; Aspinwall, E. (2005). An empirical study of the important factors for knowledgemanagement adoption in the SME sector. Journal of Knowledge Management, v. 9, n. 3, 64-82. https://doi.org/10.1108/13673270510602773

Zhang, Y. (1999). Development of structured framework for core competence evaluation in manufacturing and service industries (Doctoral theses). $\underline{U R L}$ 